\title{
Enfermedad de chagas: afección cardiaca
}

\author{
Chagas' heart disease
}

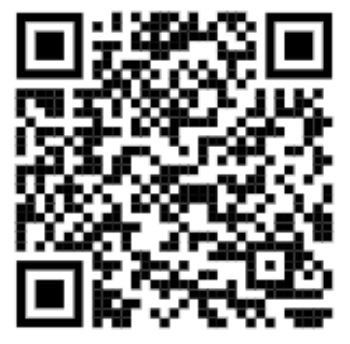

${ }^{1}$ Médico general, graduado de la Universidad de Costa Rica (UCR). Código médico: 15872 kevin.hines19@gmail.com

${ }^{2}$ Médico general, graduada de la Universidad de Costa Rica (UCR). Código médico: 15865 rebezv@gmail.com

${ }^{3}$ Médico general, graduada de la Universidad de Costa Rica (UCR). Código médico: 15820 valeriacastroco@gmail.com

\author{
${ }^{1}$ Dr. Kevin Daniel Hines Chaves \\ Investigador independiente, San José, Costa Rica \\ kevin.hines19@gmail.com \\ https://orcid.org/0000-0002-6949-3179 \\ ${ }^{2}$ Dra. Rebeca Zumbado Vásquez \\ Investigadora independiente, Heredia, Costa Rica \\ rebezv@gmail.com \\ https://orcid.org/0000-0003-1681-1871 \\ ${ }^{3}$ Dra. Valeria Castro Corrales \\ Investigadora independiente, San José, Costa Rica \\ valeriacastroco@gmail.com \\ https://orcid.org/0000-0001-7044-0675
}

RECIBIDO

CORREGIDO

ACEPTADO

$30 / 03 / 2019$

05/04/2019

$16 / 04 / 2019$

\section{RESUMEN}

La enfermedad de Chagas es una patología infecciosa producida por el parásito Trypanosoma cruzi. Debido a su carga estimada de enfermedad es considerada la enfermedad parasitaria más importante en occidente. T. cruzi es trasmitido principalmente de manera vectorial y su principal vector es Triatoma dimidiata. Cuando T. cruzi infecta a su hospedero este destruye las células infectadas y provoca una respuesta inflamatoria en diferentes órganos y tejidos. Debido a la suceptibilidad miocárdica y su baja capacidad de regeneración, el corazón es de los principales órganos implicados. La cardiopatía chagásica crónica (CCC) es la complicación más severa de la enfermedad, con gran diversidad de manifestaciones, desde infección asintomática hasta insuficiencia cardiaca con miocardiopatía dilatada y muerte súbita. Para su diagnóstico se utilizan diferentes técnicas según sea la fase en la que se encuentre la enfermedad. Asimismo, se emplean estudios de gabinete para su evaluación, con el fin de caracterizar de manera precisa la afección cardiaca. El objetivo del tratamiento es el control de la insuficiencia cardiaca y prevención de fenómenos tromboembólicos. Además para el tratamiento contra la infección como tal, se cuenta con nifurtimox y benznidazol, medicamentos a los que se les ha probado su eficacia contra $T$. cruzi. 
PALABRAS CLAVE: Trypanosoma cruzi; enfermedad de Chagas; cardiomiopatías; Triatoma; nfermedades cardiacas.

\section{ABSTRACT}

Chagas disease is an infectious disorder caused by the protozoan parasite Trypanosoma cruzi. Due to its estimated burden of disease it is considered the most important parasitic disease in the West. T. cruzi is transmitted mainly through a vector, its main vector is Triatoma dimidiata. When T. cruzi infects its host, it destroys the cells it infects, causing an inflammatory response that affects different organs and tissues. Due to the myocardium's susceptibility and its low regeneration capacity, the heart is one of the main implicated organs. Chronic Chagasic Heart Disease is the most severe complication of the disease, with a great range of manifestations, going anywhere from an asymptomatic infection up to heart failure with dilated myocardiopathy and sudden death. To diagnose this disease, there are different techniques that can be used according to the phase of the disease. In addition, studies are used for evaluation, in order to accurately characterize the heart condition. The treatment goal is to control the heart failure and prevent thromboembolic events. Specifically, for the treatment against infection, nifurtimox and benznidazole have been proven effective against $\mathrm{T}$. cruzi.

KEYWORDS: Trypanosoma cruzi; Chagas disease; cardiomyopathies; Triatoma; heart diseases.

\section{INTRODUCCIÓN}

La enfermedad de Chagas es una patología infecciosa producida por el parásito protozoario Trypanosoma cruzi (1). También conocida como Tripanosomiasis americana, la enfermedad de Chagas es la tercera parasitosis más común a nivel mundial, únicamente por detrás de la malaria y la esquistosomiasis (2); asimismo es considerada la enfermedad parasitaria más importante en occidente, con una carga estimada de enfermedad (años de vida ajustados por discapacidad), 7,5 veces mayor que la de la malaria (1).

Debido a su distribución epidemiológica es más estudiada y conocida en Latinoamérica, aspecto que se ha modificado por la globalización. Es común que cuando se mencione la enfermedad de Chagas quien haya escuchado de ella previamente recuerde el clásico signo de Romaña y quizás el insecto que la transmite, como le puede suceder a varios de los lectores (¿recuerda usted el nombre del vector?), sin embargo son menos conocidas otras de sus manifestaciones clínicas y complicaciones. El objetivo de esta revisión bibliográfica es ahondar en la patogenia y complicaciones de esta enfermedad, principalmente en su consecuencia más relevante, la miocardiopatía, la cual ocurre en 20 a $30 \%$ de las personas infectadas $(1,3)$. Se decide revisar este tema con el fin de elevar el nivel de conciencia sobre la patología, debido a que a pesar de su 
relevancia clínica, es una enfermedad que muchas veces es ignorada o desatendida por el personal médico.

\section{EPIDEMIOLOGÍA}

Los vectores más importantes viven en grietas de paredes de barro y en techos de paja de casas rurales. Los habitantes de casas infestadas se exponen repetidamente al vector y al parásito durante muchos años. En un escenario endémico la transmisión continua resulta en un aumento en la prevalencia tanto de infección como de cardiomiopatía (1).

Es endémico en ciertas áreas rurales de latinoamérica, sin embargo por la migración se ve en ciudades de latinoamérica y otros países, hasta en Japón donde se ha descrito incluso enfermedad de Chagas congénita (4). Debido a diferentes medidas de control, como el "screening" serológico en los bancos de sangre, especialmente en lugares endémicos, ha habido una disminución significativa en los últimos años en prevalencia global (18 millones en 1991 a 5.7 millones en 2010) (1).

La incidencia estimada más alta es de $4 \%$ al año en la zona hiperendémica del Chaco Boliviano, donde se encuentran poblados con una prevalencia de hasta $96.7 \%$ en personas mayores a 30 años $(1,5)$. Costa Rica se encuentra en una zona de baja prevalencia $(0.1-0.9 \%)(1,3)$, sin embargo se han descrito casos, desde infección asintomática hasta incluso de miocarditis y miocardiopatía dilatada, como un caso de diagnóstico postmortem descrito por la Dra. Moreno y colaboradores (6).

Debido a los bajos niveles de conciencia entre los proveedores de atención médica, los casos de miocardiopatía causados por enfermedad de Chagas son poco reconocidos. Sumado a esto el carácter muchas veces silencioso de la parasitosis, provoca que muchos pacientes fallezcan sin haber determinado el causante de su enfermedad $(1,6)$.

\section{ETIOLOGÍA Y PATOGÉNESIS}

T. cruzi es transmitido principalmente por las heces infectadas de un vector a través de una picadura 0 a través de las mucosas del huésped. Otras vías de transmisión son las transfusiones, trasplante de órganos o médula ósea, o congénita $(1,7,8)$. Es considerado que en Latinoamérica la transmisión vectorial es la que tiene un mayor impacto, y es la resonsable de mantener el ciclo de vida del parásito (7).

¿Recordó el lector el nombre del vector de T. cruzi? El principal vector es Triatoma dimidiata, un insecto hematófago de la subfamilia Triatominae $(1,2,7,9)$. Previamente en Centroamérica también fue relevante el vector $R$. prolixus, sin embargo debido a medidas de control de los diferentes países se logró practicamente eliminar (7). Se han empleado estrategias para eliminar a $\mathrm{T}$. dimidiata en Centroamérica como el programa de tres fases aplicado en El Salvador, Honduras, Guatemala y Nicaragua durante 2000-2015, no obstante, no han tenido éxito (7).

El nicho ecológico de T. dimidiata es muy diverso, incluye desde hábitats silvestres hasta domésticos $(7,10)$. La infestación doméstica por este vector se relaciona con paredes sin pavimentar, presencia de animales, factores ambientales como la temperatura, la humedad, el patrón de las tierras de cultivo y la vegetación (7). 
El ciclo de vida de T. cruzi se extiende desde que $T$. dimidiata ingiere tripomastigotes de la sangre de huésped infectado y posteriotmente en varias etapas se transforma en tripomastigotes metacíclicos, los cuales son su forma infecciosa y se excretan en las heces. Los tripomastigotes metacíclicos luego son transmitidos a otro o al mismo huésped mediante una picadura o a través de membranas mucosas. Estos ingresan al citoplasma de las células del hospedador y se replican en forma de amastigotos, luego vuelven a su forma de tripomastigotes, la célula se rompe y estos se liberan a la circulación con la capacidad de infectar nuevas células e iniciar nuevamente su ciclo de vida $(1,2)$. La infección de las células musculares cardiacas, macrófagos, neuronas y tejido de soporte nervioso (células gliales) pueden llegar a producir las lesiones más destacadas de esta enfermedad (6).

Posterior a la transmisión del parásito por el vector el periodo de incubación es de 1 a 2 semanas (1). Luego de este periodo ocurre una fase aguda y posteriormente una fase crónica. Durante la fase aguda la mortalidad es inferior al 5\% (6) y esta se caracteriza por ser una parasitemia microscópicamente detectable. En esta fase los síntomas suelen ser leves e inespecíficos, y estos son fiebre (continua o intermitente), cefalea, mialgias, artralgias, astenia y náuseas. Asimismo puede cursar con hepatoesplenomegalia, linfadenopatías, chagoma (proceso inflamatorio local) y el signo de Romaña (edema prolongado del párpado no doloroso) (1,6,11). La mayoría de infecciones agudas de enfermedad de Chagas no son detectadas. En menos del $1 \%$ de los casos la fase aguda es severa y amenazante para la vida (1) y estos se deben a que al producirse la ruptura celular se genera una intensa respuesta inflamatoria que lleva a miocarditis aguda, destrucción de ganglios autónomos del tracto gastrointestinal y a meningoencefalitis (6).

Luego de 4 a 8 semanas de fase aguda la respusta inmnune mediada celularmente controla la replicación, los síntomas ceden y se pasa a la fase indeterminada de la enfermedad. En esta fase no se presentan síntomas y no hay anomalidades electrocardiográficas. Un $70-80 \%$ de la población infectada permanece en esta fase por el resto de su vida. El $20-30 \%$ restante de la población infectada progresa a la forma determinada de la fase crónica, en la que se pueden presentar patologías como miocardiopatía, megaesófago, alteraciones neurológicas, y formas mixtas; principalmente las dos primeras $(1,11)$. Ver TABLA 1.

La mortalidad asociada a la enfermedad de Chagas ocurre principalmente por alteraciones eléctricas y falla cardiaca asociada a miocardiopatía dilatada (12). Debido a esto la miocardiopatía es considerada su consecuencia más importante (1) y en este artículo se decide utilizar un apartado para el desarrollo de este tema.

\section{CARDIOPATÍA CHAGÁSICA CRÓNICA}

La afección cardiaca es la manifestación más severa de la enfermedad de Chagas crónica. Se presenta años o décadas posteriores a la infección inicial. Se le conoce como cardiopatía o cardiomiopatía chagásica crónica (CCC), 
TABLA 1 Fase crónica puede tomar dos rumbos

\begin{tabular}{|c|c|c|}
\hline \multirow[t]{2}{*}{$\begin{array}{l}\text { Fase crónica } \\
\text { puede tomar dos } \\
\text { rumbos }\end{array}$} & $\begin{array}{l}20-30 \% \\
\text { Progresión a forma } \\
\text { determinada }\end{array}$ & $\begin{array}{l}\text { Progresión de la enfermedad a miocardiopatía crónica o Chagas } \\
\text { gastrointestinal o ambos. } \\
\text { Miocardiopatía: Primeros signos son defectos en conducción, } \\
\text { especialmente BRDHH o bloqueo fascicular ant. izq. La } \\
\text { miocardiopatía es altamente arritmogénica y se caracteriza por } \\
\text { bradicardia sinusal y de unión, FA o flutter, bloqueo AV y taquicardia } \\
\text { ventricular. Eventualmente se da progresión a la miocardiopatía } \\
\text { dilatada y a ICC. En etapas avanzadas se pueden dar la formación } \\
\text { de aneurismas y eventos tromboembólicos por formación de } \\
\text { trombos en VI. } \\
\text { Gastrointestinal (menos frecuente): daño de neuronas } \\
\text { intramurales que afectan esófago, colon, o ambos. Desde trastornos } \\
\text { de motilidad asintomáticos a megaesófago severo con múltiples } \\
\text { síntomas, o megacolon con fecaloma, volvulus e isquemia } \\
\text { intestinal. Más frecuente en regiones del sur de Sudamérica que en } \\
\text { regiones del norte y en Centroamérica. }\end{array}$ \\
\hline & $\begin{array}{l}70-80 \% \\
\text { forma } \\
\text { indeterminada }\end{array}$ & Sin signos ni síntomas. EKG sin anormalidades. \\
\hline \multicolumn{3}{|c|}{$\begin{array}{l}\text { Fuente: tabla original, elaborada con datos de: (1) Bern C. Chagas' Disease. New England Journal of Medicine [Internet]. } \\
2015 \text { Jul } 30 \text { [citado } 2019 \text { Marzo 27];373(5):456-66; (11) Pérez Yanez LM, Gutiérrez López A, Rodríguez Blanco S, Sarduy } \\
\text { AG. Enfermedad de Chagas. Amenaza en sombras para los corazones de la América Latina. Revista Cubana de Medicina } \\
\text { [Internet]. } 2017 \text { Jan [citado } 2019 \text { Mar 28];56(1):50-68. }\end{array}$} \\
\hline
\end{tabular}

y esta es una enfermedad progresiva que se caracteriza por una respuesta inmune inflamatoria continua que conduce a daño miocárdico y fibrosis (13).

Al producirse la infección y la lisis de células miocárdicas se desarrolla una respuesta inmunológica contra T. cruzi mediante activación de células fagocíticas y citotóxicas y producción de citocinas tipo Th1, esta respuesta protege al huésped durante la fase aguda, sin embargo, a la cronificación se asocia al daño tisular en el miocardio por una respuesta inflamatoria exacerbada principalmente con linfocitos $T$ (9).

La CCC puede presentar diferentes manifestaciones clínicas en el corazón, desde formas asintomáticas hasta enfermedad cardiaca severa, como insuficiencia cardiaca y muerte súbita (13). Respecto a estas manifestaciones, la insuficiencia cardiaca es la forma de presentación clínica más frecuente y la muerte súbita cardíaca es la mayor causa de muerte (55-65\% de los casos) (11).

En la CCC, la lesión característica es miocarditis difusa con infiltrados linfocíticos, con presencia de inmunoglobulinas con escasos elementos parasitarios y pequeños focos inflamatorios. Se produce hipertrofia de los miocitos con abundante fibrosis, que ocasiona remodelación ventricular con dilatación ventricular bilateral, con el lado derecho del corazón más dilatado que el izquierdo en la mayoría de los casos. El adelgazamiento de las paredes ventriculares es común, así como los aneurismas apicales, de la pared posterior del ventrículo izquierdo y el desarrollo de trombos murales. Se produce un deterioro de la función diastólica inicialmente y posteriormente sistólica $(9,11)$. 
Además, se presentan anormalidades de la conducción en más del $50 \%$ de los casos. La asociación de bloqueo de rama derecha y bloqueo fascicular anterior izquierdo es uno de los signos más tempranos y ocurre en $25 \%$ de los pacientes. La necesidad de utilizar un marcapasos debido a bradicardia afecta hasta un $14 \%$ de los pacientes y se presenta en cualquier fase de la enfermedad, causado por enfermedad del nodo sinusal o bloqueos AV (13).

Asimismo, se produce una inflamación periganglionar de neuronas del sistema nervioso parasimpático con adhesión de células mononucleares que provocan lisis, lo cual sumado a la reacción autoinmunitaria neuronal, condicionan la disfunción autonómica. Las alteraciones neurogénicas pueden generar muerte súbita, así como arritmias malignas y afección de ambos ventrículos que conducen a la depresión de la función ventricular global y finalmente a la cardiomegalia (9).

Además de las alteraciones de remodelación, autonómicas y de conducción descritas previamente, en la CCC se presentan anomalías microvasculares que conducen a isquemia miocárdica, mediante mecanismos que generan incremento en la adhesión plaquetaria al endotelio microvascular, disfunción endotelial, presencia de microtrombos y tendencia a espasmos (9) (11).

Se han realizado estudios en los que parece ser que la patología cardiaca puede ser causada por infección transitoria repetida, más que por persistencia local continua (12). Se ha demostrado en modelos experimentales que la re exposición al parásito en diferentes ocasiones provoca mayor cantidad de cambios y con mayor severidad en el electrocardiograma en comparación con el ser infectado una única vez (1). Asimismo, se ha evidenciado que el corazón no es infectado de forma preferente en comparación con otros tejidos, y que la susceptibilidad particular del corazón a progresar a una enfermedad amenazante para la vida se puede deber a la baja capacidad de regeneración del miocardio, al mimetismo molecular entre T. cruzi y autoantígenos del corazón, y la especial importancia de la homeostasis neurológica del corazón. Los mismos investigadores afirman que independientemente de los mecanismos por los que se produce la afección cardiaca, T. cruzi es el causante de la fibrosis miocárdica, pero que este no tiene que estar presente en el corazón de forma continua (12).

Diferentes estudios sugieren que la CCC conlleva un peor pronóstico en comparación con otras cardiomiopatías, como la idiopática, isquémica e hipertensiva $(13,14)$, y en particular el sexo masculino se ha asociado con mayor mortalidad (14). Las razones por las que su pronóstico es peor no se encuentran del todo esclarecidas (14), e incluso en investigaciones se ha demostrado que la función endotelial microvascular está igualmente deteriorada en los pacientes con CCC y cardiomiopatía isquémica (15). En un meta-análisis y revisión sistemática sobre la mortalidad en la enfermedad de Chagas se concluyó que esta enfermedad se asocia con un exceso de mortalidad estadísticamente significativo, que afecta a todos los pacientes que la padecen 
independientemente de la presentación clínica (16).

\section{DIAGNÓSTICO}

Para su diagnóstico en la fase aguda, este se realiza mediante la demostración de la presencia del parásito. La detección únicamente es posible mediante la visualización en un examen directo fresco, frotis, gota gruesa o microhematocrito, y en algunos centros mediante medios de cultivo e inoculación de animales de laboratorio (9).

En la fase crónica, debido a la baja parasitemia, se deben emplear otros métodos de detección, como lo son el ELISA (enzimoinmunoanálisis de adsorción) o IFI (inmunofluoresencia indirecta) para comprobar la presencia de anticuerpos específicos en contra de $T$. Cruzi. El diagnóstico se confirma con ambas pruebas postivas, en caso de ser discordantes los resultados se requiere realizar una tercera técnica $(2,9)$.

Como estudios de gabinete, se le debe realizar un EKG, placa PA de tórax y ecocardiograma transtorácico (ECO) a todos los paciente con enfermedad de Chagas independientemente de su edad y si presentan 0 no sintomatología cardiovascular. Estos estudios se realizan en búsqueda de evidencia de cardiomegalia (índice cardiotorácico $>0.5$ en la radiografía PA de tórax por ejemplo), derrame pericárdico, insuficiencia cardiaca y anormalidades en la conducción eléctrica del corazón. En el ECO los parámetros que se alteran inicialmente son la prolongación del tiempo de contracción y relajación isovolumétrica (9). Además se pueden apreciar alteraciones en la motilidad parietal, presencia de aneurismas y demás patologías estructurales y funcionales descritas en la patogenia de la CCC.

La resonancia magnética ( $\mathrm{RM})$ cardíaca es el examen de elección para evaluar el corazón en los pacientes con enfermedad de Chagas (17). Se ha utilizado ampliamente para la caracterización de la CCC $(17,18,19)$ debido a que proporciona imágenes de alta resolución y no utiliza radiación ionizante (17). Se han utilizado otras técnicas para evaluar la función cardiaca en esta patología, entre ellas se incluyen el marcado del miocardio; cineresonancia magnética de alta resolución y realce tardío del miocardio (para la detección de fibrosis miocárdica) (17).

\section{TRATAMIENTO}

Con respecto al tratamiento de la miocardiopatía, el objetivo es el control de la insuficiencia cardiaca y prevención de fenómenos tromboembólicos. Se recomienda el uso de betabloqueadores y de inhibidores de la enzima convertidora de angiotensina en caso de disfunción ventricular moderada o grave con fracción de eyección inferior a 40\%. En el caso de las disrritmias, se utilizan antiarrítmicos como amiodarona y en caso de que sean bradiarritmias se debe tener precaución con los betabloqueadores, bloqueadores de canales de calcio y digitálicos. Muchas de las bradiarritmias por CCC son candidatas para implantación de marcapasos. Para la prevención de tromboembolia pulmonar, se deben seguir las guías habituales para decidir si se requiere anticoagulación; no se ha definido que papel cumplen los antiagregantes plaquetarios en la 


\begin{tabular}{|c|c|c|}
\hline & \multicolumn{2}{|c|}{ Benznidazol } \\
\hline Descripción & \multicolumn{2}{|c|}{$\begin{array}{l}\text { Primera línea de tratamiento. Mejor perfil de efectos adversos que nifurtimox, y mayor } \\
\text { evidencia de eficacia. }\end{array}$} \\
\hline Dosis & \multicolumn{2}{|c|}{$\begin{array}{l}\text { edad } \\
<12: 5-7.5 \mathrm{mg} / \mathrm{kg} \mathrm{c} / \mathrm{d} \text { VO dividido en dos dosis por } 60 \text { días } \\
>12: 5-7 \mathrm{mg} / \mathrm{kg} \mathrm{c} / \mathrm{d} \text { VO en dividido en dos dosis por } 60 \text { días }\end{array}$} \\
\hline $\begin{array}{l}\text { Efectos } \\
\text { adversos }\end{array}$ & $\begin{array}{l}\text { - } \quad \text { Brotes dermatológicos leves que } \\
\text { usualmente responden a antihistamínicos. } \\
\text { - } \quad \text { Parestesia } \\
\text { - } \quad \text { Neuropatía periférica }\end{array}$ & $\begin{array}{l}\text { - } \quad \text { Anorexia/pérdida de peso } \\
\text { - } \quad \text { Náusea y vómitos } \\
\text { - } \quad \text { Leucopenia } \\
\text { - Trombocitopenia }\end{array}$ \\
\hline & \multicolumn{2}{|c|}{ Nifurtimox } \\
\hline Descripción & \multicolumn{2}{|c|}{$\begin{array}{l}\text { Nitrofurano, inhibe la síntesis de ácido pirúvico y altera el metabolismo de carbohidratos de } T \text {. } \\
\text { cruzi. }\end{array}$} \\
\hline Dosis & \multicolumn{2}{|c|}{$\begin{array}{l}\text { edad } \\
<10: 15-20 \mathrm{mg} / \mathrm{kg} \mathrm{c} / \mathrm{d} \text { dividido en } 3-4 \text { dosis por } 90 \text { días } \\
\quad 11-16: 12.5-15 \mathrm{mg} / \mathrm{kg} \text { c/d dividido en 3-4 dosis por } 90 \text { días } \\
>17: 8-10 \mathrm{mg} / \mathrm{kg} \mathrm{c} / \mathrm{d} \text { dividido en } 3-4 \text { dosis por } 90 \text { días }\end{array}$} \\
\hline $\begin{array}{l}\text { Efectos } \\
\text { adversos }\end{array}$ & $\begin{array}{l}\text { - } \text { Anorexia/pérdida de peso } \\
\text { - } \quad \text { Váuseas } \\
\text { - } \quad \text { Disconitos } \\
\text { - } \quad \text { Cefalea } \\
\text { - } \\
\quad \text { Mareos o vértigo }\end{array}$ & $\begin{array}{l}\text { - } \text { Cambios de humor } \\
\text { - Insomnio } \\
\text { - Mialgia } \\
\text { - Neuropatía periférica } \\
\text { - } \quad \text { Memoria a corto plazo disminuida } \\
\text { - Leucopenia }\end{array}$ \\
\hline
\end{tabular}

Fuente: tabla original, elaborada con datos de (1) Bern C. Chagas' Disease. New England Journal of Medicine [Internet]. 2015 Jul 30 [citado 2019 Marzo 27];373(5):456-66.

prevencion de embolias pulmonares (9). En cuanto al tratamiento de la propia enfermedad de Chagas, nifurtimox y benznidazol son los únicos fármacos con eficacia probada contra la infección por T. cruzi. Estos no se encuentran aprobados por FDA pero se pueden obtener de CDC para uso investigativo (1). Se describen en la TABLA 2.

\section{CONCLUSIÓN}

Según la bibliografía consultada, la enfermedad de Chagas es una enfermedad parasitaria de gran importancia, especialmente en Latinonamérica, y es por su frecuencia y carga de enfermedad estimada, el primer lugar de las enfermedades parasitarias en occidente. Se han realizado diferentes esfuerzos para su erradicación, y aunque se ha logrado disminuir su incidencia y prevalencia en gran medida en los últimos años, la enfermedad de Chagas continúa siendo causa de morbimortalidad en los países latinoamericanos, incluido Costa Rica.

Con la revisión realizada se planteó describir a profundidad la complicación más relevante, por su severidad, de la 
enfermedad de Chagas, la cual es la Cardiopatía Chagásica Crónica, que afecta el corazón en su aspecto estructural, autonómico, funcional y de conducción eléctrica. Se ahondó en la patogenia de la enfermedad y sus consecuencias clínicas a largo plazo, con lo cual se espera haber logrado el objetivo propuesto, al ampliar y refrescar el conocimiento sobre la afección cardiaca provocada por T. cruzi, para que el personal de salud esté atento a esta patología en la práctica clínica.

Consideramos que es especialmente relevante la intervención activa en áreas endémicas de la enfermedad, como áreas rurales con las características descritas previamente, donde habita el vector. Sin embargo, no se debe desatender en otras áreas debido a los fenómenos de migración entre países y rural-urbanos.

La dificultad en el control de esta patología se debe en parte a su gran diversidad de manifestaciones clínicas, que al poder presentarse de manera asintomática favorece a la propagación de la enfermedad, especialmente en poblaciones que se encuentran expuestos de manera repetida a la infección por el parásito.

Asimismo la detección temprana de la infección es fundamental para lograr dar un seguimiento adecuado para controlar y evitar su progresión a formas graves de la enfermedad.

\section{REFERENCIAS}

1. Bern C. Chagas' Disease. Longo DL. New England Journal of Medicine. 201507 30;373(5):456-466. https://doi.org/10.1056/nejmra1410150

2. Rivero I. Enfermedad de Chagas. Revista Medica De Costa Rica Y Centroamerica. (2016); 83 (619): 297 301p. Disponible en: http://revistamedicacr.com/index.php/rmcr/article/view/134/0

3. Lewis MD, Francisco AF, Taylor MC, Jayawardhana S, Kelly JM. Host and parasite genetics shape a link betweenTrypanosoma cruziinfection dynamics and chronic cardiomyopathy. Cellular Microbiology. 201605 25; 18(10):1429-1443 p. https://doi.org/10.1111/cmi.12584

4. Imai K, Maeda T, Sayama Y, Mikita K, Fujikura Y, Misawa K, Nagumo M, Iwata O, Ono T, Kurane I, Miyahira Y, Kawana A, Miura S. Mother-to-Child Transmission of Congenital Chagas Disease, Japan. Emerging Infectious Diseases. 2014 01;20(1):146-148. https://doi.org/10.3201/eid2001.131071

5. Clark EH, Sherbuk J, Okamoto E, Jois M, Galdos-Cardenas G, Vela-Guerra J, Menacho-Mendez GS, BozoGutierrez RW, Fernandez AB, Crawford TC, Colanzi R, Gilman RH, Bern C, . Hyperendemic Chagas Disease and the Unmet Need for Pacemakers in the Bolivian Chaco. Debrabant A. PLoS Neglected Tropical Diseases. 201406 05;8(6):e2801. https://doi.org/10.1371/journal.pntd.0002801

6. Moreno-Medina E, Valerio-Campos I, Goyenaga-Castro P. Miocarditis y miocardiopatía dilatada por Trypanosoma cruzi: Reporte de un caso. Parasitología latinoamericana. 2007 Dec; 62(3-4): 148 -153 p. https://doi.org/10.4067/s0717-77122007000200008

7. Yoshioka K, Provedor E, Manne-Goehler J. The resilience of Triatoma dimidiata: An analysis of reinfestation in the Nicaraguan Chagas disease vector control program (2010-2016). Oliveira PL. PLOS ONE. 201808 23;13(8):e0202949. https://doi.org/10.1371/journal.pone.0202949

8. Silva-dos-Santos D, Barreto-de-Albuquerque J, Guerra B, Moreira OC, Berbert LR, Ramos MT, Mascarenhas BAS, Britto C, Morrot A, Serra Villa-Verde DM, Garzoni LR, Savino W, Cotta-de-Almeida V, Meis JD. Unraveling Chagas disease transmission through the oral route: Gateways to Trypanosoma cruzi

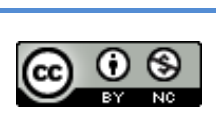


infection and target tissues. Tanowitz HB. PLOS Neglected Tropical Diseases. 2017 04 05;11(4):e0005507. https://doi.org/10.1371/journal.pntd.0005507

9. Salazar-Schettino PM, Bucio-Torres Ml, Cabrera-Bravo M, de Alba-Alvarado MC, Castillo-Saldaña R, Zenteno-Galindo E, et al. Enfermedad de Chagas en México. Revista de la Facultad de Medicina de la UNAM [Internet]. 2016 May - Junio [citado 2019 Mar 27]; 59(3): 6-16. Disponible en: http://search.ebscohost.com.ezproxy.sibdi.ucr.ac.cr:2048/login.aspx?direct=true\&db=a9h\&AN=116613805 \&site=ehost-live\&scope $=$ site

10. Gómez-Palacio A, Arboleda S, Dumonteil E, Townsend Peterson A. Ecological niche and geographic distribution of the Chagas disease vector, Triatoma dimidiata (Reduviidae: Triatominae): Evidence for niche differentiation among cryptic species. Infection, Genetics and Evolution. 2015 Dec;36:15-22. https://doi.org/10.1016/i.meegid.2015.08.035

11. Pérez Yanez LM, Gutiérrez López A, Rodríguez Blanco S, Gil SA. Enfermedad de Chagas. Amenaza en sombras para los corazones de la América Latina. Revista Cubana de Medicina [Internet]. 2017 Jan [citado 2019 Mar 28]; 56(1): 50-68 p. Disponible en: http://www.medigraphic.com/cgi$\underline{\mathrm{bin} / \text { new/resumen.cgi?IDARTICULO }=77870}$

12. Pinazo M, Lacima G, Elizalde J, Posada E, Gimeno F, Aldasoro E, Valls M, Gascon J. Characterization of Digestive Involvement in Patients with Chronic T. cruzi Infection in Barcelona, Spain. PLoS Neglected Tropical Diseases. 201408 21; 8(8): e3105. https://doi.org/10.1371/journal.pntd.0003105

13. Peixoto GDL, Martinelli Filho M, Siqueira SFD, Nishioka SAD, Pedrosa AAA, Teixeira RA, Costa R, Kalil Filho R, Ramires JAF. Predictors of death in chronic Chagas cardiomyopathy patients with pacemaker. International Journal of Cardiology. 2018 01; 250: 260-265. https://doi.org/10.1016/i.ijcard.2017.10.031

14. Assunção AN, Jerosch-Herold M, Melo RL, Mauricio AV, Rocha L, Torreão JA, Fernandes F, lanni BM, Mady C, Ramires JAF, Kalil-Filho R, Rochitte CE. Chagas' heart disease: gender differences in myocardial damage assessed by cardiovascular magnetic resonance. Journal of Cardiovascular Magnetic Resonance. 2016 Nov 28; 18: 88. https://doi.org/10.1186/s12968-016-0307-5

15. Borges JP, Mendes FDSNS, Lopes GDO, Sousa ASD, Mediano MFF, Tibiriçá E. Is endothelial microvascular function equally impaired among patients with chronic Chagas and ischemic cardiomyopathy?. International Journal of Cardiology. 2018 08; 265: 35-37. https://doi.org/10.1016/.i.jcard.2018.04.133

16. Cucunubá ZM, Okuwoga $O$, Basáñez M, Nouvellet $P$. Increased mortality attributed to Chagas disease: a systematic review and meta-analysis. Parasites \& Vectors. $2016 \quad 01$ 27; 9: 42. https://doi.org/10.1186/s13071-016-1315-x

17. Marchiori E, . Chagas disease: a tropical infection of interest to the radiologist. Radiologia Brasileira. 2016 Dec; 49(6): 5-6. https://doi.org/10.1590/0100-3984.2016.49.6e1

18. Lee-Felker S, Thomas M, Felker E, Traina M, Salih M, Hernandez S, Bradfield J, Lee M, Meymandi S. Value of cardiac MRI for evaluation of chronic Chagas disease cardiomyopathy. Clinical Radiology. 2016 06; 71(6): 618.e1-618.e7. https://doi.org/10.1016/i.crad.2016.02.015

19. Torreão JA, lanni BM, Mady C, Naia E, Rassi CH, Nomura C, Parga JR, Avila LF, Ramires JAF, Kalil-Filho $\mathrm{R}$, Rochitte CE. Myocardial tissue characterization in Chagas' heart disease by cardiovascular magnetic resonance. Journal of Cardiovascular Magnetic Resonance. 2015 Nov 18; 17: 97. https://doi.org/10.1186/s12968-015-0200-7 